\title{
UTILIZAÇÃO DA ANÁLISE DE CAOS NO PROCESSAMENTO DE SINAIS: DISCUSSÃO SOBRE PROPRIEDADES CAÓTICAS
}

\author{
H. L. MOURA ${ }^{1}$, G. J. CASTILHO ${ }^{1}$, M. A. CREMASCO ${ }^{1}$ \\ ${ }^{1}$ UniversidadeEstadual de Campinas, Faculdade de Engenharia Química \\ *e-mail: Helder_cdc@hotmail.com
}

\begin{abstract}
RESUMO
Sinais caóticos são sinais cujo comportamento é aperiódico e sensível a condições iniciais ou a pequenas perturbações. Sob este aspecto, diversos campos do conhecimento, tais como engenharia, biologia, economia, podem fazer uso da análise de séries temporais para descrever situações características a seus estudos. Contudo, encontram-se dificuldades para classificar sistemas dinâmicos caóticos a partir de métodos tradicionais como, por exemplo, o espectro de potência de Fourier. Para uma nova abordagem, a reconstrução de atratores, a partir do método de Takens, vem sendo apontada como alternativa de análise para diferenciar o caos determinístico de um comportamento estocástico. A partir do caos determinístico, várias propriedades, conhecidas como invariantes caóticas, podem ser determinadas, como a dimensão de correlação (fractal) e a entropia de Kolmogorov. O presente trabalho objetiva discutir as principais propriedades envolvidas na análise de caos e aplicações na engenharia química, tendo como base a abordagem de conceitos sobre decomposição em sistema singulares (para reconstrução do espaço de fase); dimensão de imersão; comprimento da janela; dimensão de correlação e a entropia de Kolmogorov.
\end{abstract}

\section{INTRODUÇÃO}

Muitos dos fenômenos complexos observados em experimentos nas ciências exatas são modelados por conceitos de sistema estocásticos (análises estatísticas e análises no domínio da frequência) (FERRARA E PRADO, 1994). No entanto, um grande número de trabalhos que empregam a ciência do caos para modelar vários fenômenos vem sendo utilizada, como por exemplo, em diversas áreas da engenharia de processos: Ellis et al., 2003; Manyele et al., 2006; Wu et al., 2007; Ajbar et al., 2009; Wang et al., 2010; Cremasco et al., 2011 e Castilho, 2011. A maioria desses trabalhos baseia-se em dados escalares de séries temporais para descrever fenômenos complexos advindos de uma dinâmica não linear. Nestes casos, em que nem as relações matemáticas, nem as relações das variáveis que influenciam são conhecidas, a teoria do caos fornece uma alternativa para uma caracterização qualitativa (espaço de fase) e quantitativa (dimensão de correlação e entropia de Kolmogorov) superior à abordagem tradicional estocástica (JAYAWARDENA E GURUNG, 2000). Este trabalho tem por finalidade apresentar uma revisão da aplicação da teoria do caos na análise qualitativa e quantitativa de uma série temporal experimental.

\section{RECONSTRUÇÃO DO ESPAÇO DE FASE}

Umas das formas de reconstrução do espaço de fase a partir do teorema de Takens, 
proposto por Takens (1981), é o método de atrasos (MOD). De uma forma geral pode-se representar os vetores de atraso por $\mathbf{x}_{\mathbf{i}}=\left[\mathrm{x}\left(\mathrm{t}_{\mathrm{i}}\right), \mathrm{x}\left(\mathrm{t}_{\mathrm{i}}+\tau\right), \mathrm{x}\left(\mathrm{t}_{\mathrm{i}}+2 \tau\right), \ldots, \mathrm{x}\left(\mathrm{t}_{\mathrm{i}}+(\mathrm{m}-\right.\right.$ 1) $\tau)$ ], a partir da série temporal $x_{i}=x\left(t_{i}\right), i=$ $1, . ., \mathrm{N} \quad\left\{\mathrm{x}_{i} \in R^{n \geq 2 m+1}\right\}$, no qual m é chamado de dimensão de imersão e $\tau$ é o passo de reconstrução ou tempo de atraso ("time-delay"). De acordo com Takens (1981) a reconstrução do atrator no espaço de imersão deve possui as mesmas propriedades do atrator original desde que $n \geq 2 m+1$. A Figura 1 mostra, por exemplo, uma reconstrução, a partir do teorema de Takens, para uma sequência de vetores de atrasos com $\mathrm{m}=2$ e $\tau=1$.

Existem várias dificuldades na aplicação do método dos atrasos, como por exemplo, exigir uma série temporal contínua e livre de ruídos. O teorema de Takens não faz contato direto com o processo de medição. Em particular, existem várias escalas de tempo que são especificadas, como por exemplo, o tempo de amostragem. O espaço de imersão, segundo Takens (1981), exige que $n \geq 2 \mathrm{~m}+1$. Isso, no entanto é de pouca relevância prática, visto que a priori m não é conhecido (Broomhead e King, 1986).

Figura 1 - Reconstrução bidimensional, $\mathrm{m}=2$, a partir do método de Takens (1981) com vetores sucessivos, $\tau=1$.
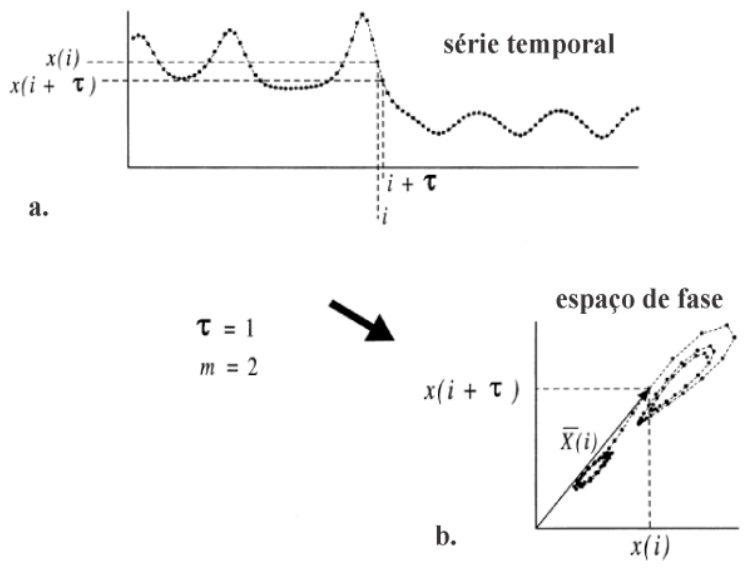

Fonte: adaptado de Johnsson et al., 2000.
O método proposto por Broomhead e King (1986), aplicado ao teorema de Takens, utiliza-se das propriedades da matriz de covariância para indicar o grau de independência para cada variável, visando obter coordenadas descorrelacionadas. A vantagem do método apresentado por Broomhead e King (1986) é justamente a eliminação do ruído, combinações simultâneas de várias frequências, contido na série temporal. Quando a projeção é realizada, uma análise dos componentes principais indicam os estados de maior variância, representando a dinâmica pura do sistema em questão, separando dos de menor variância, representando o ruído na série temporal. Os autovalores da matriz de covariância fornecerem as instruções e o grau de extensão do atrator, gerando uma melhor base para projeção de uma trajetória livre de ruídos.

\subsection{Método de Broomhead e King (1986)}

Seja a matriz de trajetória $\mathbf{X}=\left[\mathbf{x}_{1}, \mathbf{x}_{2}\right.$, $\left.\ldots, \mathbf{x}_{\mathrm{N}}\right]^{\mathrm{T}}$. Nesse caso, $\mathbf{x}_{\mathbf{i}}$ é um vetor formado por $\mathrm{N}$ elementos da série temporal e $\mathbf{x}_{\mathbf{i}+\mathbf{1}}$ é formado pelos $\mathrm{N}$ elementos seguintes dessa mesma série. As colunas da matriz de trajetória constituem os vetores de estado $\mathbf{x}_{\mathbf{i}}$ na trajetória reconstruída no espaço de imersão $\mathrm{R}^{n}$. Construídos $\mathrm{N}$ vetores de estado no espaço de imersão, procura-se encontrar um conjunto de vetores linearmente independentes $\left\{\mathbf{s}_{\mathrm{i}}\right\}$ em $\mathrm{R}^{n}$ que descreva eficientemente o espaço de fase. Esses vetores constituem parte de uma base ortonormal completa $\left\{\mathbf{c}_{\mathbf{i}}, \mathrm{i}=1,2,3, \ldots, n\right\}$ em $\mathrm{R}^{n}$ e pode ser construída como uma combinação linear dos vetores na trajetória usando a relação: $\mathbf{s}_{\mathrm{i}}^{\mathrm{T}} \mathbf{X}=$ $\sigma_{i} \mathbf{c}_{i}{ }^{\mathrm{T}}$, em que $\mathbf{S}_{\mathrm{i}}$ e $\mathbf{c}_{\mathrm{i}}$ são autovetores e $\sigma_{\mathrm{i}}$ são autovalores.

A ortonormalidade dos vetores $\left\{\mathbf{c}_{i}\right\}$ impõe que $\mathbf{s}_{\mathrm{i}} \mathbf{X} \mathbf{X}^{\mathrm{T}} \mathbf{s}_{\mathrm{j}}=\sigma_{\mathrm{i}} \sigma_{\mathrm{j}} \delta_{\mathrm{ij}}$, em que $\delta_{\mathrm{ij}}$ é o delta de Kronecker, ou seja, se $\mathrm{i}=\mathrm{j}$ implica $\delta_{\mathrm{ij}}$ $=1$ caso contrário se $\mathrm{i} \neq \mathrm{j}$ implica $\delta_{\mathrm{ij}}=0$. A matriz de estrutura $\mathbf{X} \mathbf{X}^{\mathrm{T}}$ é simétrica real, e, portanto, seus autovetores formam uma base 
ortonormal completa em $\mathrm{R}^{n}$. A partir do teorema de decomposição em valores singulares, pode ser provado que os vetores $\mathbf{S}_{\mathrm{i}}$ e $\mathbf{c}_{\mathrm{i}}$ são autovetores da matriz de estrutura $\mathbf{X} \mathbf{X}^{\mathrm{T}}$ e da matriz de covariância $\mathbf{X}^{\mathrm{T}} \mathbf{X}$ desde que $\mathbf{X} \mathbf{X}^{\mathrm{T}} \mathrm{S}_{\mathrm{i}}=\sigma_{\mathrm{i}}^{2} \mathbf{S}_{\mathrm{i}}$ e $\mathbf{X}^{\mathrm{T}} \mathbf{X} \mathbf{c}_{\mathrm{i}}=\sigma_{\mathrm{i}}^{2} \mathbf{c}_{\mathrm{i}}$, implicando que o número de autovalores diferentes de zero da matriz de estrutura é igual ao número de autovalores diferentes de zero da matriz de covariância. Isto é, posto de $\mathbf{X X}^{\mathrm{T}}=$ posto de $\mathbf{X}^{\mathrm{T}} \mathbf{X}=\mathrm{n}^{\prime} \leq \mathrm{n}$.

A decomposição dos valores singulares de $\mathbf{X}$ pode ser escrita como $\mathbf{X}=\mathbf{S D C}^{\mathrm{T}}$ no qual $\mathbf{S}=\left[\mathbf{s}_{1}, \mathbf{s}_{2}, \ldots, \mathbf{s}_{\mathrm{n}}\right], \quad \mathbf{C}=\left[\mathbf{c}_{1}, \mathbf{c}_{2}, \ldots, \mathbf{c}_{\mathrm{n}}\right] \mathrm{e}$ $\mathbf{D}=\operatorname{diag}\left[\sigma_{1}, \sigma_{2}, \ldots, \sigma_{\mathrm{n}}\right]$ com a condição que $\sigma_{1} \geq \sigma_{2} \geq \ldots \geq \sigma_{\mathrm{n}} \geq 0$. O subespaço n'- dimensional é gerado por um conjunto $\left\{\mathrm{s}_{\mathrm{i}} \mid \mathrm{i}=1, \ldots, \mathrm{n}^{\prime}\right\}$, tal que cada média correspondente sobre o $\mathbf{x}_{\mathbf{i}}$ forma uma base vetorial $\mathbf{c}_{\mathbf{i}} \in \mathrm{R}^{\mathrm{n}}$. Desse modo, o conjunto completo de $\mathbf{S i}$ atua como uma base para a construção de todas as médias possíveis sobre a trajetória. Isto é, apenas as médias associadas ao subespaço n'dimensional geram um vetor não trivial em $\mathrm{R}^{\mathrm{n}}$. Portanto, pode-se supor que o número n', o posto da matriz de covariância, tem a dimensionalidade do subespaço que contém o atrator original.

Para o efeito do ruído experimental, encontrado nas séries temporais, Broomhead e King (1986) levam em conta a análise dos autovalores e seus espectros associados. Considerando a base ortogonal $\mathbf{C}$ tem-se que $(\mathbf{X C})^{\mathrm{T}}=(\mathbf{X C})=\mathbf{D}^{2}$. A matriz $\mathbf{X C}$ é a matriz da trajetória projetada na base $\left\{\mathbf{c}_{\mathbf{i}}\right\}$. Este resultado expressa o fato de que na base $\left\{\mathbf{c}_{\mathbf{i}}\right\}$ os componentes da trajetória são não correlacionados uma vez que os $\left\{\mathbf{c}_{\mathbf{i}}\right\}$ são obtidos a partir da diagonalização da matriz de covariância. Esse resultado também mostra que cada $\sigma_{\mathrm{i}}^{2}$ é a projeção média da trajetória para os $\left\{\mathbf{c}_{\mathbf{i}}\right\}$ correspondentes. Portanto, o espectro $\left\{\sigma_{\mathrm{i}}{ }^{2}\right\}$ tem informações sobre à medida que a trajetória explora o espaço de imersão. A presença de ruído tende a mascarar o comportamento determinístico.
Em função disso, o posto da matriz de covariância n' é um limite superior para a dimensionalidade do subespaço explorado pelo componente determinístico da trajetória. Gibson et al. (1992) fazem uma análise mais simples: para uma série temporal contaminada com ruídos o espectro singular (conjunto dos autovalores não nulos da matriz de covariância) diminui até atingir certo patamar no qual os autovalores são aproximadamente iguais. Uma explicação para uma possível formação desse patamar é a presença de ruído Gaussiano na série temporal. Caso assuma-se que a série temporal $\mathrm{x}(\mathrm{t})$ é da forma $x(t)=\tilde{x}(t)+\eta(t)$, ou seja, a soma de uma série suave com um processo de ruído Gaussiano, então o ruído isotrópico, em coordenadas de atraso, impõe um limite inferior de $\left(\eta^{2}\right)$ para cada autovalor. Nesta hipótese, a altura do patamar indica a variância do ruído, e um autovalor no patamar representa o ruído-dominante no componente principal $\left(\sigma_{\mathrm{i}}\right)$. Se os componentes principais i formam uma imersão e $\sigma_{\text {i-1 }}^{2}>\eta^{2}$, o espaço de fase dos componentes principais é aproximadamente determinístico.

Ainda sobre a matriz XC, a partir do fato que os componentes da trajetória são descorrelacionados isso implica que o tempo de atraso pode ser desconsiderado. Porém, o comprimento da janela (quantidade de elementos da série presentes em cada vetor de imersão $-\tau_{\mathrm{w}}$ ) deve ser levado em conta.

\subsection{Escolhas dos parâmetros (m e $\tau_{\mathrm{w}}$ ) para o método SVD}

Em termos práticos, a dimensão de imersão m segundo Broomhead e King (1986) pode ser estimada com a quantidade de autovalores não nulos acima do patamar de ruído no espectro singular, como discutido na seção anterior.

Apesar das incertezas a respeito da determinação de $\tau_{\mathrm{w}}$, para séries temporais experimentais, algumas considerações podem ser feitas, como por exemplo, $\tau_{\mathrm{w}}$ não deve ser 
tão grande a ponto de os elementos da primeira e última janela de tempo tornar-se praticamente não correlacionados, todavia $\tau_{\mathrm{w}}$ deve ser suficientemente grande para cobrir a frequência dominante do movimento dinâmico no sistema. Para abordagens suaves do atrator, o tempo de atraso fixado em $1 / \mathrm{fs}$ (onde fs é a frequência de amostragem), o tempo entre dois pontos sucessivos na série temporal, fornece uma medida robusta para o comprimento da janela (VAN DER BLEEK E SCHOUTEN, 1993).

Para uma série temporal contínua e livre de ruídos, a implementação do método da informação mútua proposto por Fraser e Swinney (1986) para determinação do tempo de atraso, numa tentativa de associação com o comprimento da janela, fornece resultados satisfatórios. No entanto, para aplicações práticas, esse método não se mostra adequado. Estudos feito por Kugiumtzis (1996) questionam sua validade, relatando que sua natureza é empírica e não necessariamente proporciona estimativas adequadas para a reconstrução do espaço de fase com dados reais (geralmente ruidosos).

\section{INVARIANTES DO CAOS}

\subsection{Dimensão de correlação}

A dimensão de correlação $\mathrm{D}_{2}$ é um parâmetro estudado na análise caótica e está relacionado com o número de graus de liberdade do sistema. Verificou-se que a dimensão de correlação pode ser usada para caracterizar regimes e transições entre regimes (homogêneo até agitação turbulenta) de leitos fluidizados (Daw et al., 1990; van den Bleek e Schouten, 1993; Hay et al., 1995; Johnsson et al., 2000) e caracterização na transição de regimes em um leito circulante, de um regime homogêneo e diluído para um escoamento turbulento (CASTILHO, 2011).

$O$ conceito de dimensão clássico no qual $\mathrm{D} \in$ $\mathrm{Z}$ teve de ser expandido para o conceito de dimensão fractal ( $D \in R$ ), a fim de contemplar uma série de figuras geométricas (chamadas de fractais) cuja dimensão é fracionária (FERRARA E PRADO, 1994). Uma possível definição para dimensão fractal, que se reduz à dimensão clássica para objetos não fractais é apresentado na Equação 1, conhecido também por dimensão de Hausdorff.

$D_{0}=\lim _{\varepsilon \rightarrow \infty} \frac{\log N(\varepsilon)}{\log (1 / \varepsilon)}$

Na Equação $1 \mathrm{~N}(\varepsilon)$ é o número de hipercubos (caixas) de lado $\varepsilon$ necessário para cobrir todo o conjunto de pontos $A$, ou seja, $\mathrm{N}(\varepsilon)$ varia segundo $\varepsilon^{\text {-Do }}$ para $\varepsilon \rightarrow 0$ (Ferrara e Prado, 1994).

A definição da Equação 1 recupera o conceito euclidiano de dimensão inteira nos casos usuais e permite a sua generalização para conjuntos mais complexos. Se A é composto por um conjunto finito de pontos, $\mathrm{N}(\varepsilon)$ é constante para $\varepsilon \rightarrow 0$; como $\log (1 / \varepsilon)$ $\rightarrow \infty$ nesse limite, tem-se $\mathrm{D}_{0}=0$ ou, de outro modo, $\mathrm{N}(\varepsilon)$ constante e varia segundo $\varepsilon^{0}$ para $\varepsilon$ pequeno (pois não depende de $\varepsilon$ ).

A Equação 2 generaliza $\mathrm{o}$ conceito de dimensão fractal (chamada de dimensões de Renyi).

$$
D_{q}=\frac{1}{q-1} \lim _{\varepsilon \rightarrow \infty} \frac{\log _{2} \sum_{i=1}^{N(\varepsilon)} p_{i}^{q}}{\log _{2} \varepsilon}(\mathrm{q} \in \mathrm{R}, \mathrm{q} \neq 1)
$$

Com q = 2 tem-se a dimensão de correlação:

$$
D_{2}=\lim _{\varepsilon \rightarrow \infty} \frac{\sum_{i=1}^{N(\varepsilon)} p_{i}^{2}}{\log _{2} \varepsilon}
$$

O termo $p_{i}$ representa a probabilidade de um ponto se encontrar na i-ésima caixa de tamanho $\varepsilon$. 


\subsection{Algoritmo para o cálculo da dimensão de correlação}

$\mathrm{O}$ algoritmo para o cálculo de $\mathrm{D}_{2}$ desenvolvido por Grassberger e Procaccia (1983) é baseado em uma aproximação que facilita a sua programação. Essa aproximação afirma que:

A probabilidade de se ter dois pontos do atrator numa caixa de lado épsilon é aproximadamente igual à probabilidade de que a distância entre dois pontos seja menor que épsilon.

Define-se a integral de correlação como:

$$
C(\varepsilon)=\frac{1}{N^{2}} \lim _{N \rightarrow \infty} \sum_{\substack{i, j=1 \\ i \neq j}}^{N} \theta[\varepsilon-|\overrightarrow{x i}-\overrightarrow{x j}|]
$$

Em que $\theta(x)$ é a função de Heaviside, nesse caso $x=\left(\varepsilon-\left|\vec{x}_{i}-\vec{x}_{j}\right|\right)$, ou seja, $\mathrm{x} \geq 0$ implica que $\theta(\mathrm{x})=1$ se $\mathrm{x} \leq 0$ implica que $\theta(\mathrm{x})$ $=0$. A partir da Equação 4 a dimensão de correlação passa a ser escrita na forma:

$$
D_{2} \approx \lim _{\varepsilon \rightarrow \infty} \frac{\log _{2} C(\varepsilon)}{\log _{2} \varepsilon}
$$

A aproximação proposta pode ser aplicada também para as demais dimensões q através de uma generalização da Equação 4:

$$
C_{q}(\varepsilon)=\lim _{N \rightarrow \infty}\left\{\frac{1}{N} \sum_{\substack{i=1 \\ i \neq j}}^{N}\left[\frac{1}{N} \sum_{j=1}^{N} \theta\left(\varepsilon-\left|\vec{x}_{i}-\vec{x}_{j}\right|\right)\right]^{q-1}\right\}^{\frac{1}{q-1}}
$$

$O$ cálculo de $D_{2}$ se processa de forma análogo ao cálculo de $\mathrm{D}_{0}$, isto é, observando a região linear do gráfico $\log \varepsilon \mathrm{X} \log \mathrm{C}(\varepsilon)$ (Figura 2a).

Esse algoritmo é de mais fácil implementação que o de contagem de caixas (para o cálculo de $\mathrm{D}_{0}$ ), especialmente para dimensões mais altas, e mostra-se especialmente útil no contexto de sinais experimentais. Para o cálculo de $\mathrm{D}_{2}$ procede- se da seguinte maneira: um gráfico na escala $\log -\log$ é construído para a integral de correlação (Equação 4) em função de $\varepsilon$. Para cada dimensão de imersão obtém-se um valor de $\mathrm{D}_{2}(\mathrm{~m})$ (Equação 5) correspondente (Figura 2b). As inclinações do gráfico $\log \varepsilon X \log$ $\mathrm{C}(\varepsilon)$ serão aproximadamente iguais ao valor de $\mathrm{m}$ se $\mathrm{m}<\mathrm{D}_{2}$, convergindo (quando essa convergência existir) para o valor de $D_{2}$, ilustrado na Figura $2 \mathrm{~b}$, que corresponde ao grau de liberdade do sistema no qual o atrator foi imerso (FERRARA E PRADO, 1994).

Figura 2 - (a) Exemplo de gráfico $\log \varepsilon X \log$ $C(\varepsilon)$ para determinação da dimensão de correlação (b) gráfico de convergência para $\mathrm{D}_{2}$.
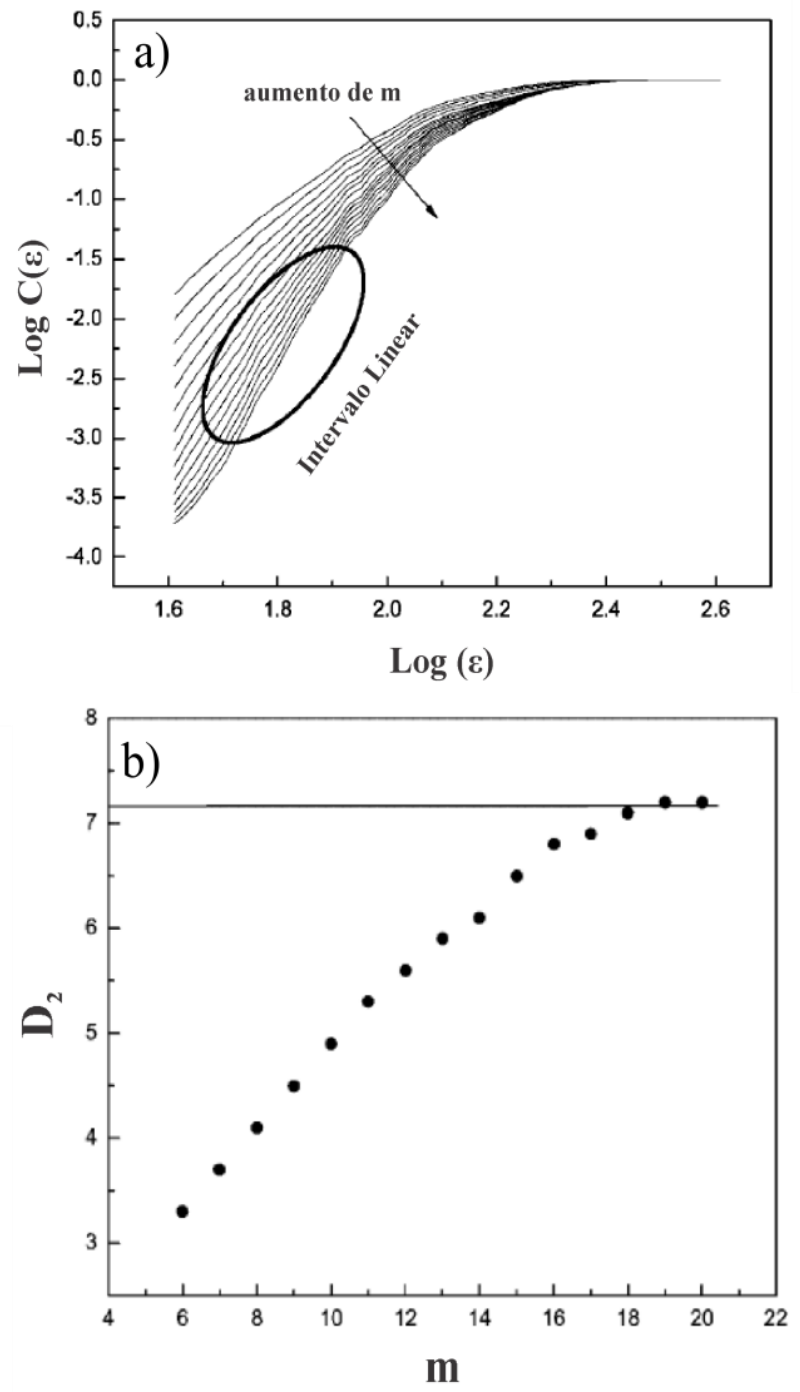

Fonte: adaptado de Xu et al., 2009. 


\subsection{Entropia de Kolmogorov}

Suponha que um evento ocorra e que seja transmitida uma mensagem relatando sua ocorrência. Define-se a quantidade de informação contida nessa mensagem como o logaritmo da razão entre a probabilidade q do evento após a mensagem ser recebida e a probabilidade $\mathrm{p}$ no receptor do evento antes da mensagem ser recebida. Num canal sem ruído, o receptor tem certeza de que a mensagem recebida está correta. Portanto, a probabilidade do evento após a mensagem ser recebida é $\mathrm{q}=1$ e a informação recebida $h$ torna-se o logaritmo do inverso da probabilidade $\mathrm{p}$, ou seja:

$$
h=\log \left(\frac{1}{p}\right)=-\log p
$$

A Equação 7 condiz com a noção de que a quantidade de informação aumenta quando a probabilidade de uma mensagem tende a zero. No outro extremo, tem-se que, quando $\mathrm{p}=1$, isto é, quando se sabe exatamente o que será transmitido, então a quantidade de informação recebida é nula $(\mathrm{h}=$ $0)$. Espera-se que o conteúdo de informação associado a duas mensagens independentes, com probabilidades de ocorrência $\mathrm{p}_{1}$ e $\mathrm{p}_{2}$, seja dado pela soma dos conteúdos de informação dessas mensagens. Ou seja, espera-se que $h\left(p_{1} p_{2}\right)=h\left(p_{1}\right)+h\left(p_{2}\right)$. Essa propriedade de aditividade ajuda a reforçar a escolha de $h$ como uma função logarítmica da probabilidade, pois $\log \left(\mathrm{p}_{1} \mathrm{p}_{2}\right)=$ $\log \left(\mathrm{p}_{1}\right)+\log \left(\mathrm{p}_{2}\right)$.

Para o caso em que há $\mathrm{N}$ mensagens alternativas, cada qual com probabilidade de ocorrência $p_{j}\left(\sum_{j} p_{j}=1\right)$ e quantidade de informação $h_{j}$, então a quantidade média de informação $\mathrm{H}$ obtida por mensagem recebida é dada por média ponderada dos $\mathrm{h}_{\mathrm{j}}$, isto é, $H=\sum_{j} p_{j} h_{j}, \mathrm{ou}$
$H=-\sum_{j=1}^{N} p j \log _{2} p j$

A Equação 8 é conhecida como entropia de Shannon ou entropia informacional, sua unidade de medida é bits por mensagem (MONTEIRO, 2006).

Considere agora um intervalo $\mathrm{b} \tau \mathrm{e}$ $(b+1) \tau,(b \in Z)$. A entropia de Kolmogorov $K$ é definida como a taxa média de criação de informação no sistema para um passo $\tau$ e lado do cubo $\varepsilon$ pequeno com uma série temporal muito longa, isto é:

$$
K=-\lim _{\tau \rightarrow 0} \lim _{\varepsilon \rightarrow 0} \lim _{b \rightarrow \infty} \frac{1}{b \tau} H
$$

Substituindo a Equação 8 na Equação 9:

$$
K=-\lim _{\tau \rightarrow 0} \lim _{\varepsilon \rightarrow 0} \lim _{b \rightarrow \infty} \frac{1}{b \tau} \sum_{j=1}^{N} p j \log _{2} p j
$$

A Equação 10 tem unidade de bits/s (FERRARA E PRADO, 1994).

Para um sistema dissipativo ordenado (determinístico), a entropia de Kolmogorov é igual à zero. Este tipo de sistema é completamente previsível em qualquer intervalo de tempo, dado o estado desse sistema e o início do intervalo. Para um sistema estocástico a entropia de Kolmogorov é igual infinito. Uma quantidade infinita de informações é necessária para prever o sistema durante qualquer intervalo, então, a localização do sistema no espaço de fase é imprevisível, mesmo após um intervalo de tempo curto. Para um sistema caótico, a entropia de Kolmogorov é finita. Isto significa que um sistema caótico somente é previsível até certo ponto dentro de um intervalo de tempo limitado (VAN DE BLEEK E SCHOUTEN, 1993).

A entropia de Kolmogorov mede a previsibilidade e a sensibilidade às pequenas perturbações do escoamento caótico: quanto 
maior a entropia, maior o grau de desorganização ou imprevisibilidade do sistema. Uma entropia de Kolmogorov positiva e finita é condição necessária e suficiente para a existência do caos em um sistema dinâmico, sendo assim fundamental a sua determinação (SHOUTEN et al., 1994).

\subsection{Algoritmo para o cálculo da entropia de Kolmogorov}

Para o algoritmo da entropia o método a seguir é baseado no trabalho de Grassberger e Procaccia (1983). Seu cálculo é baseado no recobrimento do atrator por cubos de lado $\varepsilon$ semelhante ao cálculo da dimensão de correlação. Define-se a probabilidade de uma trajetória percorrer uma dada sequencia de

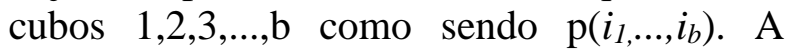
entropia de Kolmogorov é então definida como a taxa média de criação de informação no sistema entre os tempos b $\tau$ e $(b+1) \tau$, ou seja,

$$
\begin{aligned}
& K=-\lim _{\tau \rightarrow 0} \lim _{\varepsilon \rightarrow 0} \lim _{b \rightarrow \infty} \frac{1}{b \tau} \sum_{i_{1} \ldots i_{b}}^{N} p(i 1, \ldots, i b) \ln p\left(i_{1}, \ldots, i_{b}\right)= \\
& =\lim _{\varepsilon \rightarrow 0} \lim _{\Delta t \rightarrow \infty} \frac{I(\varepsilon, \Delta t)}{\Delta t}
\end{aligned}
$$

No caso de séries temporais o tempo escolhido entre as observações é fixo $(\tau=1)$ e o limite $(\tau \rightarrow 0)$ é omitido. Generalizando a definição da entropia de Kolmogorov:

$$
K_{q}=-\lim _{\tau \rightarrow 0} \lim _{\varepsilon \rightarrow 0} \lim _{b \rightarrow \infty} \frac{1}{b \tau} \frac{1}{(q-1)} \ln \sum_{i 1 \ldots i b}^{N}\left[p\left(i_{1}, \ldots, i_{b}\right)\right]^{q}
$$

$\mathrm{O}$ procedimento de cálculo se assemelha ao discutido na seção 3.2 para a obtenção de $\mathrm{D}_{2}$. Inicialmente obtém-se $\mathrm{K}_{2}$ de (11) e também a partir da generalização de (12):

$K_{2}=-\lim _{\tau \rightarrow 0} \lim _{\varepsilon \rightarrow 0} \lim _{b \rightarrow \infty} \frac{1}{b \tau} \ln \sum_{i}^{N} p_{i}^{2}=\lim _{\varepsilon \rightarrow 0} \lim _{\Delta t \rightarrow \infty} \frac{I(\varepsilon, \Delta t)}{\Delta t}$
Observando a aproximação feita na seção 3.2 , ou seja, $\sum_{i} p_{i}^{2} \sim C(\varepsilon)$ e a igualdade (13) obtém-se, no limite $\varepsilon \rightarrow 0$ e $\Delta t \rightarrow \infty$, a seguinte relação :

$$
I(\varepsilon, \Delta t)=\Delta t K_{2}(\varepsilon) \sim \ln C(\varepsilon, \Delta t)
$$

No qual $\Delta \mathrm{t}=\mathrm{b} \tau$. Reescrevendo a entropia como a diferença entre as informações em um instante de tempo $\Delta \mathrm{t}=(\mathrm{b}$ $+1) \tau$ e o seu precessor $\Delta \mathrm{t}=\mathrm{b} \tau$ obtém-se a seguinte relação a partir de (14):

$$
\tau K_{2} \sim \ln \frac{C((b+1) \tau, \varepsilon)}{C(b \tau, \varepsilon)}
$$

A entropia de Kolmogorov mostra-se ser sensível e robusta para caracterização, por exemplo, da dinâmica de leitos fluidizado e colunas de bolhas gás-líquido e pode ser usada para determinar as diferenças no comportamento dinâmico para diferentes condições de funcionamento (VAN DEN BLEEK E SCHOUTEN, 1993 e CASTILHO, 2011).

\section{CONCLUSÃO}

Neste trabalho foram apresentados alguns dos conceitos do uso da teoria do caos para uma caracterização qualitativa e quantitativa de sinais advindos de sistemas complexos. Em particular para sistemas particulados de natureza caótica, leitos fluidizados de uma forma geral, essa ferramenta mostra-se útil, como por exemplo, para detecção e caracterização de regimes, controle e monitoramento. Apresentou-se o método de reconstrução do espaço de fase utilizando o método da decomposição em valores singulares, servindo como representação da dinâmica caótica a partir de uma série temporal, em que a dimensão de imersão e o comprimento da janela do tempo foram apresentados como principais 
parâmetros para definir a qualidade da reconstrução. Além disso explicou-se de forma detalhada a utilidade do SVD para filtração de ruídos nas séries temporais. Para uma análise quantitativa descreveram-se os conceitos e utilidades dos parâmetros invariantes do caos: a dimensão de correlação, relacionada à medida do grau de liberdade do sistema e a entropia de Kolmogorov, que representa o grau de desorganização ou imprevisibilidade do mesmo.

\section{REFERÊNCIAS}

AJBAR, A., AL-MASRY, W., ALI, E. Prediction of flow regimes Transitions in bubble columns using passive acoustic measurements, Chem. Eng. Process., v.48, p.101-110, 2009.

BROOMHEAD, D.S.; KING, G.P. Extracting qualitative dynamics from experimental data. Physica D, v.20, p. 217-236, 1986.

\section{CAStilho, G. J. Análise de Caos em Leito}

Fluidizado Circulante. 2011. Tese (Doutorado em Engenharia Química), Faculdade de Engenharia Química, Universidade Estadual de Campinas, Campinas-SP, 2011.

CREMASCO, M. A. ; CASTILHO, G.J. ; ARAGON, J. M. ; Martin, L. . Experimental fluid dynamics study in a fluidized bed by deterministic chaos analysis. Part. Sci. Technol. v. 29, p. 179-196, 2011.

DAW, C. S.; LAWKINS, W. F.; DOWNING, D. J.; CLAPP, N. E. Jr. Chaotic Characteristics of a Complex Gas-Solids Flo. Phys. Rev. A, v. 41, p. 1179-1181 1990.

ELLIS, N.; BRIENS, L.A.; GRACE, J.R.; BI, H.T.; LIM, C.J. Characteristics of dynamic behavior in gas- solid turbulent fluidized bed using chaos and wavelet analyses, Chem. Eng. J. v. 96, p. 105-116, 2003.

FERRARA, N. F., PRADO, C. P. C. Caos uma introdução. São Paulo: Edgard Blücher LTDA, 1994.

FRASER, A.M.; SWINNEY, H. Independent coordinates for strange attractors from natural information. Phy. Rev. A, v.33 , p. 11341140, 1986.

GIBSON, J.E.; FARMER, J.D.; CASDAGLI, M.; EUBANK, S. An analytic approach to practical state space reconstruction. Physica D, v. 57, p. 1-30, 1992.

GRASSBERGER, P., PROCACCIA, I. Estimation of Kolmogorov Entropy from a Chaotic Signal, Phys. Rev. A, v.28 , p. 25912593, 1983.

HAY, J. M.; NELSON, B. H.; BRIENS, C. L.; BERGOUNOU, M. A. The Calculation of the Characteristics of a Chaotic Attractor in a Gas-Solid Fluidized Bed. Chem. Eng. Sci., v. 50, p. 373-380, 1995.

JAYAWARDENA, A.W., GURUNG, A.B. Noise reduction and prediction of hydrometeorological time series: dynamical systems approach vs. stochastic approach. J. Hydrol., v. 228, p.242-264, 2000.

JOHNSSON, F.; ZIJERVELD, R.C.; SCHOUTEN, J.C.; VAN DEN BLEEK, C.M.; LECKNER, B. Characterization of fluidization regimes by time-series analysis of pressure fluctuations. Int. J. Multiphase Flow, v. 26, p.663-715, 2000.

KUGIUMTZIS, D. State space reconstruction parameters in the analysis of chaotic time series - the role of the time window length. Physica D, v. 95, p. 13-28, 1996. 
MANYELE, S.V., ZHU, J.X., KHAYAT, R.E., PÄRSSINEN, J.H. Analysis of the chaotic dynamics of a high-flux CFB riser using solids concentration measurements, China Particuology, v.4, p.136-146, 2006.

MONTEIRO, L. H.A.; Sistemas Dinâmicos.

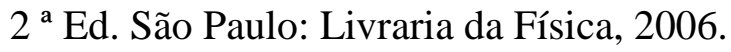

SCHOUTEN, J. C., TAKENS, F., VAN DEN BLEEK, C. M. Maximum likelihood estimation of the entropy of an attractor. Phys. Rev. E, v. 49, p. 126-129, 1994.

TAKENS, F. Detecting strange attractors in turbulence. In: Dynamical systems and turbulence, Lecture Notes in Mathematics, Eds. D.A. Rand e L-S. Young, Springer Verlag, v. 898, p. 366-381, 1981.

VAN DEN BLEEK, C. M.; SCHOUTEN, J. C. Deterministic chaos: a new tool in fluidized bed design and operation. Chem. Eng. J., v. 53, p. 75-87, 1993.

WANG, Z.Y., JIN, N.D., GAO, Z.K., ZONG, Y.B., WANG, T. Nonlinear dynamical analysis of large diameter vertical upward oilgas-watert hree-phase flow pattern characteristics, Chem. Eng. Sci., v.65, p.5526-5236, 2010.

WU, B., ZHU, J.X., BRIENS, L. A Comparison of flow dynamics and flow structure in a riser and a downer, Chem. Eng. Technol., v.30, n.4, pp.448-459, 2007.

XU, J.; BAO, X.; WEI, W.; BI, H. T.; GRACE, J. R.; LIM, C. J. Chaotic Characteristics of Pressure Fluctuations in a Gas Spouted Bed. Can. J. Chem. Eng., v. 87, p. 253-263, 2009. 